\title{
The Narcissism Expression in Publications from 1914 to 2014
}

\author{
Douglas Rossi Ramos ${ }^{1}$ \\ ${ }^{1}$ University of São Paulo (Brazil)
}

\begin{abstract}
The narcissism expression has been often used, both by common sense, as by different fields of knowledge, which demonstrates its polissemic characteristic. Thus, it is extremely important to rescue its conceptions expressed at different historical moments, for the carrying out of counterpoints about its uses and current conceptions. This research aimed to study the 'narcissism approach' at different historical moments, in its respective epistemological fields. This is a study of 'bibliographic review' on the subject and that is part of the research results of a doctoral project in psychology. The survey results showed that, at first, the 'narcissism theme' would be addressed by psychiatry and psychoanalysis; secondly, by the social sciences; and, a third moment, in correlation with elements relating to cyberspace. The study of the different historical moments in which appear the approach of narcissism has importance for a better analysis and understanding of its appropriations in the contemporary. In this sense, the study of their emergence and provenance, as well as its etymology is fundamental for its non-conceptual overestimation.
\end{abstract}

Keywords: narcissism, psychology, cyberspace.

\section{Introduction}

\subsection{Narcissism and its etymology}

The narcissism expression has been often used in the most different fields of knowledge, which demonstrates its polissemic characteristic. Thus, the study about their conceptions on different historical moments has importance for the realization of counterpoints on its uses and current conceptions.

The term narcissism has its origins in the late XIX century, from its approach in psychiatry. According Guimarães (2012), the French psychologist Alfred Binet conducted the first reference to the myth of Narcissus associated in a psychopathological problem, in 1888, from a footnote of a text on fetishism.

According Guimarães (2012), in 1899, the medical criminologist Paul Nacke write the term Narcismus (in Latin) to refer to a type of perversion. This term Narcismus (in Latin) would be consolidated in the first decade of the XX century from Krafft-Ebing, which would bring in its catalogue of sexual psychopathology.

In the same period, Sigmund Freud (2012) would use this term in Latin (Narcismus) initially in the minutes of the Psychoanalytic Society of Vienna (preceding the text 'On Narcissism: an Introduction' of the 1914). After that, the term would be translated into German until establish its final spelling such as we know it today.

From this, this article aims to present a study on the different approaches of narcissism term in different fields of knowledge. Thus, a survey was conducted in database files about the narcissism theme, published between 1914 and 2014.

The results showed that, in addition of psychiatry and psychoanalysis, the narcissism would be a topic also addressed by sociology and philosophy, and, more currently, associated with the cyberspace context.

\section{Discussion}

\subsection{The publications about narcissism in the last 100 years}

The survey was conducted in 15 databases: PsycINFO, Academic Search Complete, Business Source Complete, ERIC, Teacher Reference Center, Hospitality \& Tourism Complete, PsycARTICLES, Psychology and Behavioral Sciences Collection, GreenFILE, European Views of the Americas: 1493 to 1750, Library \& Information Science Source, Library, Information Science \& Technology Abstracts, Regional Business, Scielo Brasil. 
The search was performed using the expression 'narcissism' as reference for titles and keywords. This survey databases was conducted in 2015, and, therefore, presents texts published by the year 2014 .

The following table (table 1) presents the amount of scientific articles, books, dissertations and academic theses, published over the last ten years (2004 to 2014), in which there is the 'narcissism' expression in titles and keywords.

TABLE 1: Publications databases in which there is the term 'narcissism' during the period 2004-2014

\begin{tabular}{ccccc}
\hline \hline Themes/Publications (2004- 2014) & Scientific Articles & Books & Dissertations/Theses & Total \\
\hline Narcissism & 4448 & 365 & 214 & 5027 \\
Narcissism and Psychoanalysis & 2775 & 101 & 14 & 2890 \\
Narcissism and Psychotherapy & 506 & 51 & 16 & 573 \\
Narcissism and Psychopathology & 438 & 17 & 20 & 475 \\
Narcissism and Psychiatry & 249 & 28 & 10 & 287 \\
Narcissism and Neuroscience & 59 & 9 & 4 & 72 \\
Narcissism and Internet & 96 & 1 & 0 & 106 \\
Narcissism and Philosophy of Difference & 4 & & & 5 \\
\hline \hline
\end{tabular}

It is possible to observe in Table 1 that most of the texts and works published which there is the occurrence of the term 'narcissism', are present in the field of 'psychoanalysis'. In general, the 'narcissism theme' appears associated to publications on the 'mental health' and 'psychopathological dimensions', in addition a pejorative sense.

The works in the field of philosophy consists in texts whose theoretical framework concerns the so-called 'philosophy of difference', expressed by authors such as Gilles Deleuze and Michel Foucault. In this category, there are published works by authors such as Orlandi (2003), Cardoso Jr (2004, 2005), Alves (2007) and Henz (2009).

The novelty are, therefore, its growing association to the subject of 'Internet' and an early approach to the field of the 'philosophy of difference'.

The table 2 below presents the survey, in the same database, of the occurrence of the term 'narcissism' in works published since 1914 (the year of publication of the text 'On Narcissism: an introduction').

TABLE 2 - Occurrence of publications in which there is the term 'narcissism', during the period of 1914 until 2014

\begin{tabular}{cc}
\hline \hline Publications (1914 - 2014) & Occurrence of the 'narcissism theme' \\
\hline Scientific Articles & 8580 \\
Books & 870 \\
Dissertations/Theses & 563 \\
Total & 10013 \\
\hline \hline
\end{tabular}

Comparing the Table 1 with the Table 2, it's possible verifying that, in a period of 100 years (1914-2014), about $53 \%$ of all publications in which there is the occurrence of the term 'narcissism' were made in the last ten years. In other words, by subtracting the difference between the two tables, it's possible verifying that of 1914 until 2014 (in 100 years) were published 10013 papers that reference in the title or keywords to the term narcissism, while of 2004 until 2014 ( in the last 10 years) were published 5027 (53\%) of this total.

Evidently, one must consider that today it is easier to publish articles, mainly because there are several platforms of digital scientific journals, and that there is a greater number of universities and graduate programs today than 100 years ago. However, in all aspects, the differences are significant, because, for example, of the 870 books in which there is the occurrence of the term 'narcissism' published in the last 100 years, 505 books $(58 \%)$ corresponded to the last 10 years.

In order to get specific parameters for comparison, the next step was to conduct the search for publications in the database of a single university (University of São Paulo). In this sense, it was possible to verify would be present that the same trend of growing interest about the 'narcissism theme' (in the last 10 years).

According to this survey in databases of University of São Paulo, there was 211 published works until 2014. Of the 211 texts, 103 (49\%) correspond to works published in the last 10 years. When separating these works by type of publication, we found that, of this total, 27 publications correspond to articles in periodicals, among which 17 (63\%) were published in the last 10 years. 
Regarding the dissertations in which there is reference in titles or keywords to the term narcissism were located a total of 14 works. Of these 14 publications, almost all were published in the last 10 years, and $78 \%$ of the total (11 dissertations) correspond to publications of the last five years.

Finally, regarding the doctoral theses, there was a total of 10 works published. All these theses were published in the last six years, with half of that total (5 theses) in the last two years (2013 and 2014). It is important to emphasize that the production areas that was based these theses and dissertations were the 'psychoanalysis', the 'critical theory' and 'literature'.

Overall, these surveys show a growing interest on the narcissism theme in recent years and warn of the risk of their possible 'conceptual overestimation'.

To proceed with the analysis, the Table 3, below, shows the occurrences of the term 'narcissism' associated with the 'Internet', since the 90s, period that would begin to consolidate and popularize the World Wide Web:

TABLE 3 - Occurrence of publications in which there is the term 'narcissism' and 'narcissism and internet' over the period 1990-2014

\begin{tabular}{cc}
\hline \hline Publications (1990 a 2014) & Occurrence of the term 'narcissism' \\
\hline Narcissism & 7880 \\
Narcissism and Internet & 116 \\
\hline \hline
\end{tabular}

In this new correlation, it is noted that there is an incipient production of texts that present the rapprochement between narcissism and internet. When comparing the Table 3 with the Table 1, it is possible to see that these 116 publications, approximately 91\% (106 works) occurred in the last 10 years (period that coincides with the creation and popularization of 'Social Medias' like Facebook, as well as smartphones).

\section{Considerations}

When considering these searches in the database on the subject of narcissism, it is possible to highlight three distinct moments:

. the first refers to the late XIX century and early XX century, in which there is the approach of the 'narcissism theme' with the psychiatry and then emerging Freud's psychoanalysis;

. the second comprises the second half of the XX century, and corresponds to its appropriation by the social sciences, whose the main axis of articulation concerns the 'consumer society';

. the third corresponds to the current period, in which the question of narcissism also would gain prominence with its articulation in parallel to the popularization of the 'Social Medias' and specific uses of the mobile technologies, such as smartphones.

In the first moment of great evidence in the academic and scientific publications on the subject, reasoned from the contribution of Freud's psychoanalysis, the 'narcissism' expression is no longer a speculative term", to establish itself as 'concept' (as a element constitutive and foundational of the human psyche) .

In the second phase, there is its expansion into the field of social sciences, and under the influence of the publication of the book "The Culture of Narcissism: American Life in an Age of Diminishing Expectations" of Christopher Lasch (1991) (original version published in 1979), the expression narcissism began to be used to describe cultural aspects (Lasch is the first to publish the term 'culture of narcissism').

Finally, in the third moment, which corresponds to the current time, the term 'narcissism' would be frequently used to refer to news modalities of inter-relationships in digital spaces. In this case, it is necessary, however, be careful when invoking the term narcissism, because the modes of relationships mediated by digital technologies consist in a new paradigm to constitution of subjectivities, in which the self-presentation is a prominent feature (the issue of self-presentation in the digital should not be restricted or abridged with a 'label' stamped 'narcissism').

The study of the different historical moments in which appear the narcissism approach has importance for a better analysis and understanding of its appropriations in the contemporary. In this sense, the study of their emergence and provenances is fundamental for its non-conceptual overestimation, as, for example, on approach to the cyberspace. 


\section{References}

[1] M.F.F. Alves, "The neo-narcissism as one of the modes of subjectivity in disciplinary societies and societies of control," Final report of research, Fapesp, Dept. Psychology, Unesp, São Paulo, Brazil, 2007.

[2] H. R. Cardoso jr, "Nomadism and nomadology to contemporary subjectivities, according Deleuze and Guattari." In Social Control Strategies, L. Rocha, and S.M. França, IEEE Ed. Arts and Science, ch.4, pp. 125-133.

[3] H.R. Cardosos jr, "What a subjectivity is useful for? Foucault, Time and Body." IEEE Psicol. Reflex. Crit., vol. 18, pp. 343-349, August. 2005.

[4] S. Freud. On Narcissism: an Introduction. 4st ed. Karnac, UK: London, 2012, ch. 5, pp. 54-75.

[5] L. M. Guimarães, "Three studies on the concept of narcissism in Freudian work: origin, metapsychology and social forms" M.S. dissertation, Dept. Psychology, University of São Paulo, São

[6] A. O. Henz, "Training as deformation: exhaustion between Nietzsche and Deleuze,"IEEE Rev. Malaise Subj., vol. 9 , pp. 135-159, March 2009.

[7] C. Lasch, The Culture of Narcissism: American Life in an Age of Diminishing Expectations, 3st ed. Norton \& Company, EUA: New York, 1991, ch. 2, pp. 1-275.

[8] L.B.L. Orlandi, Skirting the Deleuzian reading of the tragic in Nietzsche., "IEEE magazine Look, vol. 7, pp. 10-26, December, 2003. 